\title{
AVANZANDO EN AUTODETERMINACIÓN: ESTUDIO SOBRE LAS AUTOPERCEPCIONES DE PERSONAS ADULTAS CON DISCAPACIDAD INTELECTUAL DESDE UNA PERSPECTIVA DE INVESTIGACIÓN INCLUSIVA
}

\section{Advancing in self-determination: study on the self-perceptions of adults with intellectual disabilities from an inclusive research perspective}

\author{
Vanessa Vega Córdova \\ Pontificia Universidad Católica de Valparaíso. Chile \\ Izaskun Álvarez-AgUAdo \\ Pontificia Universidad Católica de Valparaíso. Chile \\ izaskun.alvarez@pucv.cl \\ Herbert Spencer GonzÁLEZ \\ Pontificia Universidad Católica de Valparaíso. Chile \\ Félix González Carrasco \\ Pontificia Universidad Católica de Valparaíso. Chile
}

Recepción: 12 de abril de 2019

Aceptación definitiva: 22 de octubre de 2019

RESUMEN: La autodeterminación se ha convertido en el constructo clave para garantizar el desarrollo de habilidades que permitan a las personas con discapacidad adquirir el control sobre sus vidas. Este estudio, desde un paradigma de investigación inclusiva, busca valorar las autopercepciones sobre autodeterminación de 122 personas adultas con discapacidad intelectual en Chile. Los datos de esta investigación se han recopilado mediante un cuestionario ad hoc en formato de software online y una entrevista semiestructurada. Los principales resultados evidencian cómo los y las participantes presentan 
bajos niveles de autodeterminación. El desarrollo de competencias relacionadas con la autodefensa o autoinstrucción está muy descendido a diferencia de otras habilidades como la toma de decisiones o la resolución de problemas. A partir de estas evidencias se discute cómo los desafíos a los que se enfrentan estas personas durante su adultez (reubicación del lugar de residencia, envejecimiento, etc.) exigen nuevas estrategias que orienten el trabajo sobre autodeterminación. Además, el desarrollo de investigaciones inclusivas que involucran la participación real de personas con discapacidad intelectual se revela como un elemento importante para el fomento de la autodeterminación.

PALABRAS ClAve: discapacidad intelectual; adultez; autodeterminación; inclusión.

AвsтRAct: Self-determination has become the key construct to ensure the development of skills that allow people with disabilities to gain control over their lives. This study, from an inclusive research paradigm, seeks to assess the self-perceptions about self-determination of 122 adults with intellectual disabilities in Chile. The data of this research have been collected through an ad hoc questionnaire in online software format and a semi-structured interview. The main results show how the participants have low levels of self-determination. The development of competencies related to self-defense or self-instruction are greatly diminished unlike other skills such as decision making or problem solving. From these evidences it is discussed how the challenges that these people face during their adulthood (relocation of the place of residence, aging, etc.) demand new strategies that guide the work on self-determination. In addition, the development of inclusive research involving the real participation of people with intellectual disabilities is recognized as an important element for the promotion of self-determination.

KEY WORDs: intellectual disabilities; adulthood; self-determination; inclusion.

\section{Introducción}

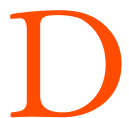

URANTE EL ÚLTIMO DECENIO la autodeterminación se ha convertido en el constructo por excelencia a favor del empowerment en el ámbito de la discapacidad (Schalock, Keith, Verdugo y Gómez, 2010; Wehmeyer et al., 2011; Wehmeyer, Palmer, Shogren, Williams-Diehm y Soukup, 2013). Paulatinamente, este concepto se ha ido concretando como una disposición personal a ejercer control sobre la propia conducta convirtiéndose en un indicador de calidad de vida y en una meta educativa prioritaria (Schalock et al., 2010). Aunque se trata de un constructo multidimensional, han sido tres las perspectivas que han adquirido mayor relevancia a la hora de operativizar el concepto. Por una parte, está el enfoque ecológico, ampliamente estudiado por autores como Abery y Stancliffe (2003). Por otro lado, el enfoque sociopolítico relaciona el constructo con los derechos civiles y los movimientos de vida independiente (Wehmeyer, Little y Sergeant, 2009). Por último, y desde el ámbito psicoeducativo, este concepto se concreta en el Modelo Funcional de Autodeterminación (Wehmeyer, 1999), que recientemente se ha redefinido bajo el paradigma de la Teoría de la Agencia Causal (Shogren et al., 2015a). 
AVANZANDO EN AUTODETERMINACIÓN: ESTUDIO SOBRE LAS AUTOPERCEPCIONES DE PERSONAS ADULTAS CON DISCAPACIDAD INTELECTUAL DESDE UNA PERSPECTIVA DE INVESTIGACIÓN INCLUSIVA V. VEGA CÓRDOVA, I. ÁLVAREZ-AGUADO, H. SPENCER GONZÁLEZ Y F. GONZÁLEZ CARRASCO

Desde este modelo, la autodeterminación se entiende como el conjunto de acciones volitivas que ayudan a la persona a actuar como agente causal primario en su propia vida sin influencias externas innecesarias (Wehmeyer, 1999). Es decir, son todas aquellas habilidades que permiten controlar aspectos importantes: defender las propias opiniones, elegir entre distintas opciones, plantear objetivos realistas, tener metas propias, etc. En el campo de la discapacidad se ha hecho especial hincapié en comprobar la incidencia tanto de elementos contextuales (Arellano y Peralta, 2015; Grigal, Neubert, Moon y Graham, 2003) como de factores personales en el logro de la autodeterminación (Carter, Lane, Pierson y Stang, 2008; Gómez-Vela, Verdugo, González, Badía y Wehmeyer, 2012; Nota, Ferrari, Soresi y Wehmeyer, 2007; Shogren, Wehmeyer, Palmer y Paek, 2013).

En cuanto a la incidencia de factores contextuales, se ha identificado cómo el estilo de crianza (Wehmeyer et al., 2011) o la estructura familiar (Vicente, Guillén, Verdugo y Calvo, 2018) condicionan el desarrollo de la autodeterminación. Del mismo modo, las evidencias sobre la influencia de los entornos educativos formales en el desempeño de habilidades de autodeterminación sugieren que el sistema de creencias de los y las profesionales incide en su desempeño (Grigal et al., 2003). Igualmente, la intensidad de los apoyos (más o menos frecuentes) que reciben los y las estudiantes con discapacidad (Vicente et al., 2017) y las oportunidades que ofrece el entorno escolar para trabajar la autodeterminación (Mumbardó-Adam et al., 2017) se revelan como elementos explicativos de la misma.

Con respecto a la influencia de factores personales en el logro de la autodeterminación, la literatura evidencia resultados diversos. Mientras en algunos estudios la autodeterminación se ve condicionada por el grado de discapacidad, la edad o el género (Nota et al., 2007; Shogren et al., 2013), en otras investigaciones el desarrollo de habilidades para la autodeterminación está más influenciado por variables como la conducta adaptativa o las habilidades sociales (Carter et al., 2008; Gómez-Vela et al., 2012).

No obstante, si bien los resultados de estas investigaciones son dispares, los autores coinciden en identificar tres factores influyentes en el desarrollo de habilidades para la autodeterminación: las competencias personales, las oportunidades que ofrece el entorno y los apoyos contextuales. Atendiendo al progresivo avance sobre el estudio de estos tres elementos, la literatura internacional muestra suficientes indicios por los que promover la autodeterminación se considera una buena práctica: las personas con discapacidad intelectual tienen mayor participación en la comunidad (Wehmeyer et al., 2011), mejor reconocimiento de las fortalezas individuales (Wehmeyer et al., 2013), relaciones sociales más positivas (Schalock et al., 2010) y su inclusión laboral es más satisfactoria (Wehmeyer et al., 2011). Además, en el ámbito educativo, trabajar habilidades de autodeterminación en el aula facilita la participación de estudiantes con discapacidad en actividades dentro del currículum ordinario (Agran, Cavin, Wehmeyer y Palmer, 2010; Kim y Park, 2012; Lee, Wehmeyer, Palmer, Soukup y Little, 2008), incrementa la adquisición de competencias relacionadas con la resolución de problemas (Agran, Blanchard, Wehmeyer y Hughes, 2002; Cote et al., 2014) y favorece el establecimiento y la consecución de metas personales (Finn, Getzel y McManus, 2008; Kleinert, Harrison, Dueppen, Mills y Tailor, 2014). 
AVANZANDO EN AUTODETERMINACIÓN: ESTUDIO SOBRE LAS AUTOPERCEPCIONES DE PERSONAS ADULTAS CON DISCAPACIDAD INTELECTUAL DESDE UNA PERSPECTIVA DE INVESTIGACIÓN INCLUSIVA V. VEGA CÓRDOVA, I. ÁLVAREZ-AGUADO, H. SPENCER GONZÁLEZ Y F. GONZÁLEZ CARRASCO

A pesar de los buenos resultados, la investigación sobre autodeterminación más reciente sugiere ampliar el estudio del constructo. En primer lugar, los autores evidencian la necesidad de investigar el desarrollo de habilidades para la autodeterminación en personas adultas, ya que la mayor parte de los estudios sobre autodeterminación se focalizan en la adolescencia y en la etapa de transición a la vida adulta (Chadwick y Fullwood, 2018; Chou, Wehmeyer, Palmer y Lee, 2017; Frielink, Schuengel y Embregts, 2018; Shogren, Wehmeyer, Palmer, Rifenbark y Little, 2015), existiendo un gran desconocimiento sobre cómo seguir trabajando estas habilidades en etapas posteriores.

La escasa literatura sobre autodeterminación en la adultez evidencia algunas barreras para poder desarrollarla: el deterioro cognitivo propio del envejecimiento (Chadwick y Fullwood, 2018), la falta de alternativas laborales (Frienlink et al., 2018), la sobreprotección familiar (Shogren et al., 2015b), etc. No obstante, a pesar de las barreras, en algunos estudios (Jones et al., 2018; Oswald et al., 2018; Vega, ÁlvarezAguado y Jenaro, 2018; Wehmeyer, Shogren y Thompson, 2018) las personas adultas con discapacidad intelectual manifiestan tener habilidades para ser autodeterminadas y plantean deseos de superar retos. Estas investigaciones, además de poner el foco en el desarrollo de la autodeterminación durante la adultez, evidencian la importancia de estudiar el constructo a partir de las autopercepciones de las propias personas. La mayoría de las investigaciones sobre discapacidad y autodeterminación en esta etapa de la vida parten de las opiniones de heteroinformantes: familia, profesionales, etc. A juicio de Jones et al. (2018) y Oswald et al. (2018) esto supone un sesgo importante a la hora de interpretar la autodeterminación que es necesario ir erradicando.

Precisamente, el concepto de investigación inclusiva surge desde la investigación emancipatoria para garantizar la plena participación de las personas con discapacidad intelectual en investigaciones que les atañen. Autores como Walmsley, Strnadová y Johnson (2018) identifican algunas de las principales características de estas investigaciones, entre las que destacan: el tema debe ser de interés para las personas con discapacidad, el proceso debe tener un carácter colaborativo, las personas con discapacidad deben poder ejercer cierto control sobre las etapas de la investigación, todo el proceso debe ser accesible: constructos, informes, herramientas, etc. Existen varias iniciativas que han ayudado a esclarecer los roles que desempeñan las personas con discapacidad según su participación en estas investigaciones: como asesores, como colaboradores o como líderes de sus propios procesos de investigación (Salmon, Barry y Hutchins, 2018). Los resultados de estos estudios evidencian un incremento considerable del bienestar personal y un mayor desarrollo de habilidades relacionadas con la autoinstrucción, autonomía y autoevaluación en las personas con discapacidad intelectual que participan en investigaciones inclusivas (Pallisera, Fullana, Puyaltó, Vilà y Díaz, 2017; Salmon et al., 2018; Walmsley et al., 2018). De hecho, las propias personas admiten que estas experiencias les ayudan a expresarse libremente sobre asuntos que les son importantes, a defender sus derechos y a participar de actividades socialmente valoradas (Petri et al., 2018). Según Pallisera et al. (2017), el valor agregado de la investigación inclusiva pasa por el reconocimiento de las experiencias y opiniones de las personas con discapacidad intelectual. Además, es un modelo de investigación respetuoso con el enfoque de derechos que actualmente impera en este ámbito. 
Este trabajo, desde un enfoque de investigación inclusiva, busca precisamente valorar las autopercepciones sobre autodeterminación de un grupo de personas adultas con discapacidad intelectual en Chile. Hasta la fecha, en el contexto chileno se han desarrollado varias iniciativas dedicadas a fomentar la vida independiente (Castro, Casas, Sánchez, Vallejo y Zúñiga, 2016; Vega, Jenaro, Flores, Cruz y Lerdo, 2012), la accesibilidad universal (Mella, Díaz, Muñoz, Orrego y Rivera, 2016) o la calidad de vida de personas con discapacidad intelectual (Vega, Jenaro, Morillo, Cruz y Flores, 2011). Los resultados de estas investigaciones revelan algunos aspectos emergentes de los que, tal y como se ha evidenciado con anterioridad, también se ha hecho eco la literatura internacional (Chadwick y Fullwood, 2018; Oswald et al., 2018; Wehmeyer et al., 2018). Entre los aspectos más emergentes, destaca la necesidad de seguir ahondando en el estudio de las habilidades de autodeterminación que presenta este colectivo (Castro et al., 2016; Mella et al., 2016; Vega et al., 2012). Este hecho va a facilitar la definición de lineamientos e instancias formativas que permitan mejorar la adquisición y desarrollo de estas competencias a lo largo de la vida de estas personas.

\section{Método}

Se plantea un estudio de tipo mixto (cuantitativo y cualitativo), de carácter descriptivo, inferencial y transversal, que busca conocer y valorar las autopercepciones sobre autodeterminación de un grupo de personas adultas con discapacidad intelectual en el contexto chileno.

\subsection{Participantes}

La muestra fue de conveniencia y estuvo compuesta por 122 personas adultas con discapacidad intelectual cuyas edades oscilaban entre los 25 y 55 años. La mayor parte de los participantes fueron hombres $(53.3 \%)$. Al inicio de este estudio, el $68 \%$ de los y las participantes trabajaba en entornos normalizados en la Región Metropolitana y en la Región de Valparaíso (Chile). Los criterios de inclusión para la conformación de la muestra fueron ser mayor de 18 años, contar con habilidades comunicativas, participar activamente en instituciones de apoyo a la vida independiente y tener un diagnóstico de discapacidad intelectual leve o moderada. Cabe reseñar que 11 de esos 122 participantes (6 mujeres y 5 hombres) fueron seleccionados para formar un equipo asesor que trabajase ininterrumpidamente con los investigadores e investigadoras de este estudio en las diferentes fases del proceso: elaboración de los instrumentos, análisis de los datos, validación de las herramientas, diseño de la interfaz del cuestionario, etc.

\subsection{Instrumento}

Para la recopilación de los datos se diseñó un cuestionario ad hoc autodirigido. Esta herramienta se elaboró en formato de software online para dispositivos móviles. 

ADULTAS CON DISCAPACIDAD INTELECTUAL DESDE UNA PERSPECTIVA DE INVESTIGACIÓN INCLUSIVA V. VEGA CÓRDOVA, I. ÁLVAREZ-AGUADO, H. SPENCER GONZÁLEZ Y F. GONZÁLEZ CARRASCO

El proceso de validación preliminar del instrumento constó de varias fases. En primer lugar, junto con el equipo asesor se revisaron dos escalas: Escala de autodeterminación personal ARC (Wehmeyer, 1995) y Escala ARC-INICO de evaluación de la autodeterminación (Verdugo et al., 2015). Se trata de dos herramientas de tipo autoinforme dirigidas a personas con discapacidad intelectual. A pesar de estar validadas, ambas escalas presentaban una serie de características que imposibilitaban su aplicación en esta investigación. Entre las principales cracterísticas destacaron: a) la no existencia de información sobre si sus ítems se habían definido desde la accesibilidad cognitiva, siendo este un requisito indispensable para el desarrollo de investigaciones inclusivas; b) la edad mínima de los y las participantes de esta investigación era de 25 años y el rango etario al que se dirige la escala ARC-INICO es de 11-19 años; y c) la versión para personas adultas de la escala de autodeterminación personal ARC no estaba validada transculturalmente en el momento en el que se desarrolló el estudio. Partiendo de estas premisas y para construir el instrumento ad hoc se analizó la estructura de estas dos escalas y la correspondencia entre sus ítems y dimensiones. Esto permitió operacionalizar el constructo y determinar el número de ítems a considerar (Tabla 1).

\section{TABLA 1. Dimensiones, indicadores e ítems de la herramienta codiseñada}

\begin{tabular}{|l|l|l|l|}
\hline \multicolumn{1}{|c|}{ CATEGORÍA } & \multicolumn{1}{|c|}{ DIMENSIÓN } & \multicolumn{1}{c|}{ INDICADOR } & \multicolumn{1}{c|}{ EJEMPLO ÍTEM } \\
\hline Voluntad & Autonomía & $\begin{array}{l}\text { Realización de elecciones } \\
\text { Toma de decisiones } \\
\text { Resolución de problemas }\end{array}$ & $\begin{array}{l}\text { Elijo cosas en mi día a día } \\
\text { Soluciono algunos } \\
\text { problemas }\end{array}$ \\
\hline Competencias & Autorregulación & $\begin{array}{l}\text { Establecimiento de metas } \\
\text { Autoinstrucción } \\
\text { Autoevaluación }\end{array}$ & $\begin{array}{l}\text { Planifico mi tiempo libre } \\
\text { Aprendo cosas que me } \\
\text { interesan }\end{array}$ \\
\hline Opoyos & Control-eficacia & $\begin{array}{l}\text { Autodefensa } \\
\text { Locus de control interno } \\
\text { Expectativas de logro } \\
\text { Atribuciones de eficacia }\end{array}$ & $\begin{array}{l}\text { Soy capaz de dar mi } \\
\text { opinión } \\
\text { Reclamo cuando algo no } \\
\text { me gusta }\end{array}$ \\
\hline Autoconocimiento & $\begin{array}{l}\text { Sé qué cosas me hacen } \\
\text { sentir mal } \\
\text { Sé que hay cosas que me } \\
\text { cuesta hacer }\end{array}$ \\
\hline
\end{tabular}

En total se definieron 44 ítems distribuidos en cuatro dimensiones y once indicadores. Estos ítems se englobaron en cuatro categorías: voluntad, competencias, apoyos y oportunidades. Las categorías se relacionaron con aquellos factores que la literatura especializada (Wehmeyer et al., 2011) identifica como elementos que deben estar presentes para desarrollar la autodeterminación. Estas categorías fueron transversales y jerárquicamente superiores a las dimensiones de autodeterminación (autonomía, autorregulación, control-eficacia y autorrealización) y sus correspondientes indicadores desde el Modelo Funcional de Autodeterminación (Wehmeyer, 1999). Por su parte, la redacción de cada ítem se formuló en conjunto con el equipo asesor, 
de manera que todos fuesen accesibles cognitivamente para cualquier potencial usuario. Los y las participantes debieron descargar la aplicación desde sus dispositivos móviles y, en función del enunciado de los diferentes ítems, seleccionar una opción atendiendo a una escala Likert de 6 puntos desde "no puedo hacerlo aunque me apoyen" hasta "puedo hacerlo solo".

Todos los ítems fueron sometidos a juicio de cinco personas expertas para garantizar la validez de contenido del instrumento diseñado. Para determinar la confiabilidad del acuerdo interjueces se utilizó el coeficiente de validez de contenido total (Cvct) (Pedrosa, Suárez-Álvarez y García-Cueto, 2103). En conjunto otorgaron un Cvct = 0.90. Según Pedrosa et al. (2013) el parámetro considerado aceptable para asegurar la improbabilidad de un acuerdo azaroso es de Cvet $=0.58$. Por lo tanto, el uso de los ítems elaborados se consideró adecuado. En cuanto a la fiabilidad del instrumento, los análisis de consistencia interna calculando el Alpha de Cronbach arrojaron un $\alpha=$ .938. El valor de Alpha evidenció índices aceptables que superaron el rango sugerido ( $\alpha=.700)$ por Pérez-Llantada y López (2004). Los valores de Alpha para las cuatro categorías del instrumento también manifestaron altos niveles de consistencia: volun$\operatorname{tad}(\alpha=.917)$, competencias $(\alpha=.943)$, apoyos $(\alpha=.906)$ y oportunidades $(\alpha=.924)$.

Asimismo, para complementar los datos cuantitativos se aplicó una entrevista semiestructurada únicamente a los miembros del equipo asesor $(\mathrm{n}=11)$. Atendiendo a las sugerencias de Sandín (2003) la entrevista se estructuró en categorías. En este caso, las categorías fueron las mismas que en el cuestionario: voluntad, competencias, apoyos y oportunidades. Los diferentes indicadores sobre autodeterminación se fueron evaluando según esas categorías y las preguntas orientadoras para cada una de ellas (Tabla 2). Al igual que el cuestionario, la entrevista también fue sometida a tres jueces expertos, considerándola válida para su uso y posterior análisis (Cvct $=0.88)$.

\begin{tabular}{|l|l|}
\hline \multicolumn{2}{|c|}{ TABLA 2. Categorías de la entrevista, indicadores de medida } \\
y preguntas orientadoras
\end{tabular}

\subsection{Procedimiento}

Para la conformación de la muestra se contactó con tres organizaciones de atención a la discapacidad de las regiones Metropolitana y de Valparaíso (Chile). A los 
responsables de las mismas se les mencionaron el objetivo del estudio, los criterios de inclusión de la muestra y el encuadre de la investigación. En este caso, se les comentó que se trataba de un estudio enmarcado bajo el paradigma de investigación inclusiva (Walmsley et al., 2018; Walmsley y Johnson, 2003). Como se ha comentado previamente, asumir un modelo de investigación inclusiva supone garantizar la participación real de las personas con discapacidad intelectual en las distintas etapas de los procesos de investigación que les atañen (Strnadová, Cumming, Knox y Parmenter, 2014). En esta investigación se optó por acoger una de las formas que la literatura sugiere para concretar la participación real de estas personas en las investigaciones que les son propias: conformar grupos asesores con poder de decisión que trabajen colaborativamente con los investigadores e investigadoras durante todo el proceso (Bigby, Frawley y Ramcharan, 2014).

Una vez definido el equipo asesor, se fijó con ellos y ellas un encuentro inicial en dependencias de la Pontificia Universidad Católica de Valparaíso (Chile), donde se les explicitó el propósito de la investigación y los roles a desempeñar. Tras la firma de los consentimientos informados (aprobados por el comité de ética de la universidad) se establecieron encuentros semanales de trabajo (jornadas de tres horas). La ejecución de las diferentes fases de la investigación permitió consolidar algunos roles en estos procesos. Los miembros del comité asesor transitaron desde aprendices, pasando por informantes, expertos por experiencia hasta coinvestigadores (Tabla 3 ).

\begin{tabular}{|c|c|}
\hline \multicolumn{2}{|c|}{$\begin{array}{l}\text { TABLA 3. Principales roles desempeñados por el equipo asesor durante } \\
\text { el proceso de investigación }\end{array}$} \\
\hline ROL & EJEMPLO DE TAREA \\
\hline Aprendices & $\begin{array}{l}\text { Capacitarse en temas relacionados con procesos de investigación. } \\
\text { Comprender el constructo de autodeterminación y sus diferentes } \\
\text { dimensiones e indicadores. }\end{array}$ \\
\hline Informantes & $\begin{array}{l}\text { Compartir experiencias de vida relacionadas con el desarrollo de } \\
\text { habilidades para la autodeterminación. Explicitar barreras y facili- } \\
\text { tadores para el desarrollo de la autodeterminación. }\end{array}$ \\
\hline Expertos por experiencia & $\begin{array}{l}\text { Definir los ítems y las distintas opciones de respuesta del instru- } \\
\text { mento para la recolección de datos. Validar las interfaces de la apli- } \\
\text { cación informática (letra, color, tamaño, íconos, etc.). }\end{array}$ \\
\hline Coinvestigadores & $\begin{array}{l}\text { Discutir los resultados de la implementación de las herramientas y } \\
\text { analizarlos. Evaluar el proceso de investigación e identificar aspec- } \\
\text { tos de mejora y temas emergentes. }\end{array}$ \\
\hline
\end{tabular}

El trabajo con el equipo asesor constó de tres fases (Tabla 4). En la fase de capacitación, el comité asesor adquirió habilidades para abordar las distintas etapas que conforman una investigación y también se trabajó el concepto de autodeterminación. En la fase de ejecución, y una vez adquiridas las habilidades previas, el equipo asesor participó en las distintas etapas de construcción del cuestionario: definición de indicadores e ítems, validación de las interfaces y pantallas de la aplicación (forma, color, 
tamaño de la letra, etc.), análisis de los resultados de la implementación piloto, identificación de mejoras, etc. Por último, en la fase de reflexión, se evaluó el desarrollo del proyecto en su totalidad. Para la ejecución de las diferentes tareas el comité asesor obtuvo apoyo de expertos y expertas en investigación y en educación especial, así como material en lectura fácil y en formato audiovisual.

\begin{tabular}{|c|c|c|}
\hline FASES & TAREAS & ACTIVIDADES REALIZADAS POR LOS ASESORES \\
\hline \multirow[t]{2}{*}{ Capacitación } & $\begin{array}{l}\text { Solicitud del } \\
\text { comité de ética }\end{array}$ & $\begin{array}{l}\text { 1. Revisión del protocolo de consentimiento informado } \\
\text { para participar en la investigación. } \\
\text { 2. Conocimiento de las implicaciones éticas que tiene una } \\
\text { investigación. }\end{array}$ \\
\hline & $\begin{array}{l}\text { Revisión de la } \\
\text { literatura }\end{array}$ & $\begin{array}{l}\text { 1. Lectura de material sobre autodeterminación. } \\
\text { 2. Discusión sobre la definición de autodeterminación. } \\
\text { 3. Identificación de las características de la autodeterminación. }\end{array}$ \\
\hline \multirow[t]{3}{*}{ Ejecución } & $\begin{array}{l}\text { Formulación } \\
\text { de preguntas de } \\
\text { investigación }\end{array}$ & $\begin{array}{l}\text { 1. Identificación de temas de investigación emergentes so- } \\
\text { bre autodeterminación. } \\
\text { 2. Capacitación para la formulación de hipótesis y pregun- } \\
\text { tas de investigación. } \\
\text { 3. Codefinición de hipótesis y preguntas de investigación. }\end{array}$ \\
\hline & $\begin{array}{l}\text { Elección y } \\
\text { desarrollo del } \\
\text { método de } \\
\text { investigación }\end{array}$ & $\begin{array}{l}\text { 1. Formación sobre paradigmas y técnicas de investigación } \\
\text { científica. } \\
\text { 2. Identificación de las diferentes etapas de una investiga- } \\
\text { ción científica. } \\
\text { 3. Definición de las tareas a realizar en las diferentes etapas } \\
\text { de una investigación científica. } \\
\text { 4. Revisión y selección de instrumentos para evaluar auto- } \\
\text { determinación. }\end{array}$ \\
\hline & $\begin{array}{l}\text { Elaboración y } \\
\text { validación del } \\
\text { instrumento de } \\
\text { evaluación }\end{array}$ & $\begin{array}{l}\text { 1. Revisión de pasos para el diseño de instrumentos de evalua- } \\
\text { ción sobre autodeterminación (cuestionario y entrevista). } \\
\text { 2. Identificación de dimensiones e indicadores de medida. } \\
\text { 3. Construcción de ítems y preguntas para evaluar autode- } \\
\text { terminación. } \\
\text { 4. Validación preliminar de ítems y preguntas para evaluar } \\
\text { autodeterminación. }\end{array}$ \\
\hline \multirow[t]{2}{*}{ Reflexión } & $\begin{array}{l}\text { Análisis de los } \\
\text { datos }\end{array}$ & $\begin{array}{l}\text { 1. Sistematización de los datos en categorías de análisis pre- } \\
\text { viamente establecidas. } \\
\text { 2. Discusión de los resultados y extracción de las conclusio- } \\
\text { nes más relevantes tras el proceso de evaluación. }\end{array}$ \\
\hline & $\begin{array}{l}\text { Preparación } \\
\text { de informes y } \\
\text { divulgación de } \\
\text { resultados }\end{array}$ & $\begin{array}{l}\text { 1. Participación en grupos focales para valorar el proceso de } \\
\text { investigación y sus principales resultados. } \\
\text { 2. Presentación de las experiencias de investigación en ins- } \\
\text { tancias de difusión científica. }\end{array}$ \\
\hline
\end{tabular}


Para la implementación piloto del instrumento, además de la cumplimentación del mismo por parte de los 11 miembros del equipo asesor, se invitó a participar a 111 personas con discapacidad intelectual provenientes de las entidades con las que se había establecido contacto previamente. La aplicación del instrumento se realizó de manera escalonada tras la firma de los consentimientos informados. Los investigadores e investigadoras se desplazaron hasta las sedes de las instituciones para presentar la herramienta, proporcionar las instrucciones de uso y supervisar el proceso.

Se procedió a complementar la información cuantitativa mediante la aplicación de entrevistas semiestructuradas únicamente a los 11 miembros del equipo asesor. La construcción de las categorías y preguntas de la entrevista emergió de los ítems formulados para el cuestionario y fueron definidas con la colaboración del comité asesor. Se utilizó el Modelo Secuencial (Hsieh y Shannon, 2005) para estructurar el desarrollo de este proceso: definición del tema, elaboración y validación del guion y aplicación de la entrevista. La aplicación de cada entrevista osciló entre los 45-50 minutos de duración y fueron moderadas por dos de las investigadoras con formación en educación especial.

\subsection{Análisis}

En cuanto a los procedimientos utilizados para el análisis de los datos, cabe reseñar que se obtuvieron evidencias de dos fuentes: cuestionario ad hoc y entrevista semiestructurada. Los resultados derivados de la aplicación piloto del cuestionario se analizaron a través del programa SPSS-22 mediante estadísticos descriptivos, tendencias centrales y pruebas de normalidad (Kolmogorov-Smirnov) que precedieron al uso de estadísticos paramétricos (ANOVA). En cuanto al estudio de las evidencias proporcionadas por las entrevistas, tras la transcripción y validación de las mismas se efectuó un análisis de contenido temático (Sandín, 2003) con el uso del programa ATLAS.ti.

\section{Resultados}

Los resultados de la investigación se estructuran en dos apartados. Por una parte, se presentan aspectos referidos al análisis de la implementación del cuestionario. En segundo lugar, se describen las autopercepciones de los y las participantes sobre el constructo de autodeterminación desde una aproximación cualitativa mediante la aplicación de las entrevistas.

\subsection{Análisis de los datos obtenidos del cuestionario}

Para facilitar la interpretación de los resultados, las puntuaciones de la escala se han dividido en tres rangos (bajo, medio, alto). Como se observa en la Tabla 5, los 

ADULTAS CON DISCAPACIDAD INTELECTUAL DESDE UNA PERSPECTIVA DE INVESTIGACIÓN INCLUSIVA V. VEGA CÓRDOVA, I. ÁLVAREZ-AGUADO, H. SPENCER GONZÁLEZ Y F. GONZÁLEZ CARRASCO

niveles de autodeterminación de la mayoría de los y las participantes se sitúan en un rango bajo $(n=91)$. Es decir, un $74.6 \%$ de la muestra apenas ejecuta conductas autodeterminadas. Además, un $25.4 \%$ de encuestados presenta niveles medios en el desarrollo de su autodeterminación. Por otro lado, no existen participantes cuyos niveles de autodeterminación estén posicionados en un rango alto.

\begin{tabular}{|l|c|c|}
\hline \multicolumn{3}{|c|}{ TABLA 5. Distribución de las puntuaciones totales del cuestionario según rangos } \\
\hline RANGOS & FRECUENCIA & $\%$ \\
\hline Bajo & 91 & 74.6 \\
\hline Medio & 31 & 25.4 \\
\hline Alto & 0 & 0 \\
\hline
\end{tabular}

Para complementar el análisis se ha estudiado la distribución de las puntuaciones atendiendo a las cuatro dimensiones del instrumento: autonomía, autorregulación, control-eficacia y autorrealización. En la Tabla 6 se observa cómo el desarrollo de habilidades asociadas a la dimensión de control-eficacia $(\bar{\alpha}=2.86)$ está más presente entre las competencias sobre autodeterminación de la muestra. Sin embargo, el desempeño de habilidades relacionadas con la autorregulación está más descendido según las autopercepciones de los y las participantes $(\bar{\alpha}=1.87)$.

\section{TABLA 6. Estadísticos descriptivos según las dimensiones de autodeterminación}

\begin{tabular}{|l|c|c|}
\hline \multicolumn{1}{|c|}{ DIMENSIÓN } & MEDIA & DESVIACIÓN TÍPICA \\
\hline Autonomía & 2.74 & 0.732 \\
\hline Autorrealización & 2.29 & 0.734 \\
\hline Control-eficacia & 2.86 & 0.564 \\
\hline Autorregulación & 1.87 & 0.873 \\
\hline
\end{tabular}

Si se pone el foco de atención en los indicadores sobre autodeterminación asociados a las distintas dimensiones (Tabla 7), el desarrollo de habilidades de autoevaluación $(\bar{\alpha}=3.96)$, el desempeño de competencias de locus de control interno $(\bar{\alpha}=3.42)$ y el establecimiento de metas $(\bar{\alpha}=3.17)$ presentan los promedios más elevados. No obstante, según las autopercepciones de los y las participantes, el desarrollo de habilidades para la autoinstrucción $(\bar{\alpha}=1.32)$, la autodefensa $(\bar{\alpha}=1.58)$ y el autoconocimiento $(\bar{\alpha}=1.86)$ apenas se refleja en sus conductas.

\begin{tabular}{|l|c|c|}
\hline \multicolumn{2}{|c|}{ TABLA 7. Estadísticos descriptivos según indicadores de autodeterminación } \\
\hline \multicolumn{1}{|c|}{ INDICADOR } & MEDIA & DESVIACIÓN TíPICA \\
\hline Resolución de problemas & 2.42 & .495 \\
\hline Realización de elecciones & 2.90 & 1.182 \\
\hline Toma de decisiones & 2.92 & .831 \\
\hline
\end{tabular}

Ediciones Universidad de Salamanca / CC BY-NC-ND

Siglo Cero, vol. 51 (1), 2020, enero-marzo, pp. 31-52 
AVANZANDO EN AUTODETERMINACIÓN: ESTUDIO SOBRE LAS AUTOPERCEPCIONES DE PERSONAS ADULTAS CON DISCAPACIDAD INTELECTUAL DESDE UNA PERSPECTIVA DE INVESTIGACIÓN INCLUSIVA V. VEGA CÓRDOVA, I. ÁLVAREZ-AGUADO, H. SPENCER GONZÁLEZ Y F. GONZÁLEZ CARRASCO

\begin{tabular}{|l|c|c|}
\hline \multicolumn{2}{|c|}{ TABLA 7. Estadísticos descriptivos según indicadores de autodeterminación (cont.) } \\
\hline \multicolumn{1}{|c|}{ INDICADOR } & MEDIA & DESVIACIÓN TÍPICA \\
\hline Autoconocimiento & 1.86 & .873 \\
\hline Establecimiento de metas & 3.17 & .730 \\
\hline Autoinstrucción & 1.32 & .904 \\
\hline Autoevaluación & 3.96 & 1.705 \\
\hline Autodefensa & 1.58 & .903 \\
\hline Locus de control interno & 3.42 & .219 \\
\hline Expectativas de logro & 2.45 & .336 \\
\hline Atribuciones positivas de eficacia & 2.91 & 1.525 \\
\hline
\end{tabular}

Para ahondar en los resultados, se ha analizado la existencia de diferencias significativas en función de algunas variables sociodemográficas: edad, género, situación laboral y participación en el equipo asesor de esta investigación. Para ello, tras comprobar la normal distribución de los datos mediante la prueba de Kolmogorov-Smirnov, se ha procedido al cálculo del estadístico ANOVA. Al comparar las puntuaciones generales con las diferentes variables sociodemográficas, encontramos que únicamente existen diferencias significativas atendiendo a la participación de la muestra en el equipo asesor. Como se observa en la Tabla 8, existen diferencias significativas en los niveles generales de autodeterminación en función de si se participa o no en el equipo asesor $(p=.021)$. En este caso, las personas que forman parte del equipo asesor presentan un promedio significativamente más elevado en sus niveles de autodeterminación $(\bar{\alpha}=2.93)$ que aquellos que no participan en este comité $(\bar{\alpha}=2.44)$.

\begin{tabular}{|l|c|c|c|c|}
\hline \multicolumn{5}{|c|}{ TABLA 8. Diferencias significativas en la escala total atendiendo } \\
a la participación en el equipo asesor \\
\hline & SUMA DE CUADRADOS & MEDIA CUADRÁTICA & F & SIG. \\
\hline Intergrupos & 2.386 & 2.386 & 5.433 & $.021^{*}$ \\
\hline Intragrupos & 52.251 & 0.439 & & \\
\hline
\end{tabular}

* La diferencia es significativa al nivel de .005 (bilateral).

Del mismo modo, se ha estudiado si el hecho de participar o no en el equipo asesor incide en las puntuaciones asociadas a las dimensiones de autodeterminación. Como se aprecia en la Tabla 9, existen diferencias significativas en las dimensiones de autonomía $(p=.029)$, autorregulación $(p=.025)$ y autorrealización $(p=.003)$. Sin embargo, participar en el equipo asesor no indica un mayor nivel de desarrollo de habilidades de control-eficacia $(\mathrm{p}=.061)$. El análisis de los promedios de las dimensiones atendiendo a la participación de la muestra en el equipo asesor evidencia que quienes forman parte del mismo presentan puntuaciones significativamente más elevadas en las dimensiones de autonomía $(\bar{\alpha}=3.12)$, autorregulación $(\bar{\alpha}=2.76)$ y autorrealización $(\bar{\alpha}=2.61)$ que quienes no participan en el equipo asesor. 


\begin{tabular}{|l|c|c|}
\hline \multicolumn{3}{|c|}{ TABLA 9. Diferencias significativas en las dimensiones atendiendo } \\
a la participación en el equipo asesor
\end{tabular}

* La diferencia es significativa al nivel de .005 (bilateral).

Profundizando en el análisis de las puntuaciones generales asociadas a los diferentes indicadores que conforman el constructo de autodeterminación, como se observa en la Tabla 10 los puntajes de algunos de estos indicadores difieren en función de la participación de la muestra en el equipo asesor. En concreto, existen diferencias significativas a la hora de realizar elecciones $(\mathrm{p}=.014)$, tomar decisiones $(\mathrm{p}=.045)$, desarrollar habilidades para el autoconocimiento $(\mathrm{p}=.003)$, el establecimiento de metas $(\mathrm{p}=.018)$, la autoevaluación $(\mathrm{p}=.022)$ y las atribuciones positivas de eficacia $(\mathrm{p}=$ 013). En todos estos indicadores, los promedios para el grupo de personas que forman parte del comité asesor son más elevados que para aquellas personas que no participan como miembros del equipo asesor.

\begin{tabular}{|l|c|c|}
\hline \multicolumn{2}{|c|}{$\begin{array}{c}\text { TABLA 10. Diferencias significativas en los indicadores atendiendo } \\
\text { a la participación en el equipo asesor }\end{array}$} \\
\hline \multicolumn{1}{|c|}{ INDICADOR } & F & SIG. \\
\hline Resolución de problemas & 0.269 & .605 \\
\hline Realización de elecciones & 6.180 & $.014^{*}$ \\
\hline Toma de decisiones & 4.110 & $.045^{*}$ \\
\hline Autoconocimiento & 9.409 & $.003^{*}$ \\
\hline Establecimiento de metas & 5.755 & $.018^{*}$ \\
\hline Autoinstrucción & 1.379 & .243 \\
\hline Autoevaluación & 5.404 & $.022^{*}$ \\
\hline Autodefensa & 1.767 & .186 \\
\hline Locus de control interno & 1.799 & .100 \\
\hline Expectativas de logro & 0.000 & $.013^{*}$ \\
\hline Atribuciones positivas de eficacia & 6.289 & \\
\hline
\end{tabular}

* La diferencia es significativa al nivel de .005 (bilateral).

Por otro lado, se ha analizado la relación existente entre las puntuaciones generales de la muestra sobre autodeterminación y las cuatro grandes categorías de análisis en las que se estructura el cuestionario: voluntad, competencias, apoyos y oportunidades. Atendiendo a la Tabla 11, el análisis de correlación aplicando Pearson evidencia 

ADULTAS CON DISCAPACIDAD INTELECTUAL DESDE UNA PERSPECTIVA DE INVESTIGACIÓN INCLUSIVA V. VEGA CÓRDOVA, I. ÁLVAREZ-AGUADO, H. SPENCER GONZÁLEZ Y F. GONZÁLEZ CARRASCO

una relación alta y positiva entre los promedios de las cuatro categorías y el nivel medio de autodeterminación autopercibido por los participantes. El estadístico Chicuadrado permite determinar la asociación entre estas variables. En este caso, la voluntad de querer ser autodeterminado $\left(\mathrm{x}^{2}=40.523 ; \mathrm{p}=.000\right)$, las competencias que se tienen para ello $\left(x^{2}=39.490 ; p=.000\right)$, los apoyos recibidos $\left(x^{2}=40.049 ; p=.000\right)$ y las oportunidades ofrecidas $\left(\mathrm{x}^{2}=40.122 ; \mathrm{p}=.000\right)$ inciden de forma significativa en los niveles de autodeterminación autopercibidos. Es decir, el nivel de autodeterminación se incrementa cuanto mayor es la voluntad de querer ser autodeterminado o autodeterminada; mayores las competencias que se tienen para ello, y más los apoyos y oportunidades recibidas.

\begin{tabular}{|c|c|c|c|c|c|}
\hline \multicolumn{6}{|c|}{$\begin{array}{l}\text { TABLA 11. Correlación entre el promedio de las categorías } \\
\text { y la puntuación media total de la escala }\end{array}$} \\
\hline & & vo & $\mathrm{CO}$ & $\mathrm{AP}$ & OP \\
\hline \multirow[t]{2}{*}{ Escala total } & Correlación Pearson & $.969 * *$ & $.968 * *$ & $.922 \% *$ & $.936^{* *}$ \\
\hline & Sig. (bilateral) & .000 & .000 & .000 & .000 \\
\hline
\end{tabular}

vo: voluntad; CO: competencias; AP: apoyos; OP: oportunidades.

* La correlación es significativa al nivel de .001 (bilateral).

\subsection{Análisis de las evidencias proporcionadas por la entrevista}

Para complementar los resultados cuantitativos se ha aplicado una entrevista semiestructurada a los 11 miembros del equipo asesor. Los resultados de las entrevistas (E) se han estructurado atendiendo a estas categorías: voluntad, competencias, apoyos y oportunidades.

En cuanto a la voluntad para ser autodeterminado, la mayor parte de los encuestados y encuestadas considera relevante desarrollar habilidades relacionadas con la autodeterminación: "Es importante porque te hace ser el protagonista de tu vida" (E4). "Yo quiero ser autodeterminado para decidir las cosas que me importan" (E2). Las principales razones que justifican la voluntad de querer ser autodeterminado o autodeterminada tienen que ver con la sensación de control de la situación: "Siendo autodeterminado uno puede controlar lo que le pasa" (E5). "Es importante hacerlo porque así yo me controlo y pienso antes de actuar" (E6).

En cuanto a la importancia que dan a las diferentes dimensiones de la autodeterminación, coinciden en que hay mayor predisposición a aprender todas aquellas habilidades que les permitan resolver problemas (autonomía) y hacer cosas por sí solos (autorregulación): “Quiero resolver problemas por mí mismo porque generalmente mis problemas los resuelven otros” (E3). "Quiero profesionalizarme en mi área como ayudante de párvulo” (E2). "Me gustaría andar solo, viajar, vivir solo y aprender a hacer todo eso" (E4). No obstante, pese a la voluntad para desarrollar competencias de autodeterminación, los y las participantes también hacen referencia a algunos hándicaps que pueden condicionar el querer ser autodeterminado: “Aunque es importante

Ediciones Universidad de Salamanca / CC BY-NC-ND

Siglo Cero, vol. 51 (1), 2020, enero-marzo, pp. 31-52 
tengo miedo de hacerlo mal” (E8). "Me da un poco de susto porque imagínate que elijo y lo hago mal" (E9).

Con respecto a las competencias para desarrollar autodeterminación, entre los discursos de los y las participantes se observan algunas habilidades relacionadas con el constructo. Los comportamientos autodeterminados identificados tienen que ver, sobre todo, con la realización de elecciones o la ejecución de rutinas cotidianas: "Elijo la ropa que me quiero poner cada día” (E1). "Compro las cosas que a mí me gustan” (E11). "Sé ir desde mi casa hasta el trabajo sin problemas" (E5).

Los entrevistados y las entrevistadas también cuentan con habilidades para resolver problemas y autoevaluarse en distintas situaciones: "Aunque es difícil intento resolver situaciones que me complican” (E10). "Cuando voy a alguna parte y no sé llegar le pregunto a alguien si puede orientarme” (E4). "Una vez me di cuenta que cometí un error y aprendí que no debía volver a hacerlo" (E6). "Cuando hago algo siempre pienso cómo lo puedo mejorar para la próxima”. No obstante, en ocasiones admiten tener grandes dificultades para desarrollar habilidades de autodefensa: "No digo lo que pienso porque puede que los demás lo tomen mal” (E7). "No me gusta dar mi opinión porque puede que no se entienda" (E9). "Prefiero que me defiendan otras personas" (E6). "Trato de reclamar lo menos posible, soy la persona más pasiva que hay” (E3).

Por otra parte, en los relatos de los y las participantes se identifican algunos tipos de apoyo a la autodeterminación. Principalmente aluden a familiares y profesionales como principales fuentes de apoyo: "Lo importante es saber qué hacer y para eso a veces hay que pedir ayuda a la familia" (E8). "Si tengo dudas le pregunto a mi madre" (E9). "A veces pregunto a los profesionales del centro cuál es la mejor opción para mí” (E2). Aunque en menor medida, la pareja y las amistades son también figuras de apoyo a la autodeterminación: "Cuando tengo un problema lo comparto con mi pareja” (E1). "Los amigos de confianza me animan a hacer las cosas por mí mismo" (E4). No obstante, en ocasiones las personas de apoyo obstaculizan el desarrollo de habilidades para la autodeterminación: "Yo sé que soy capaz pero a veces a mi tía le da miedo" (E2). "A veces mi madre me impide controlar las cosas" (E11). "A veces mis amigos lo hacen por mí. Necesito un apoyo, un empuje, un ánimo. Pero no que lo hagan” (E10).

Por último, los discursos de los y las participantes permiten identificar elementos que facilitan o dificultan las oportunidades para desarrollar autodeterminación. En su mayoría, reconocen que apenas tienen oportunidades para llevar a cabo el tipo de vida que quieren: "No puedo trabajar donde me gustaría porque el entorno no está adaptado para personas con discapacidad" (E1). "No me dejan gestionar mi dinero" (E4). "No puedo alquilar una vivienda por tener discapacidad" (E8). "Para mí está casi prohibido tener hijos" (E5). A pesar de las limitaciones contextuales también existen algunos facilitadores: "Hay cursos que puedes hacer para tener mejor autoestima y conocerte mejor. Yo los hago” (E4). “Aquí (se refiere al equipo asesor) estoy aprendiendo a ser más autodeterminada porque me dejan opinar” (E7).

En términos generales, los y las participantes destacan más barreras que facilitadores a la hora de llevar a cabo comportamientos autodeterminados. Si bien reconocen que hay oportunidades concretas para el desempeño de este tipo de conductas, 
AVANZANDO EN AUTODETERMINACIÓN: ESTUDIO SOBRE LAS AUTOPERCEPCIONES DE PERSONAS ADULTAS CON DISCAPACIDAD INTELECTUAL DESDE UNA PERSPECTIVA DE INVESTIGACIÓN INCLUSIVA V. VEGA CÓRDOVA, I. ÁLVAREZ-AGUADO, H. SPENCER GONZÁLEZ Y F. GONZÁLEZ CARRASCO

indican que estas instancias son muy escasas en el contexto en el que se desenvuelven: "Hay poco lugar para la autodeterminación en Chile" (E1).

\section{Discusión y conclusiones}

En línea con la literatura disponible (Chadwick y Fullwood, 2018; Frielink et al., 2018; Jones et al., 2018), los resultados de esta investigación ponen de manifiesto cómo el desarrollo de habilidades para la autodeterminación es un tema aún pendiente entre el colectivo de personas adultas con discapacidad intelectual. Los datos de este estudio coinciden con las conclusiones de investigaciones como las de Frielink et al. (2018) y Oswald et al. (2018) al evidenciar niveles bajos de autodeterminación en la etapa adulta.

En concreto, y en línea con Frielink et al. (2018), entre los y las participantes se observan escasas actitudes relacionadas con el autoconocimiento o la autodefensa. Precisamente, algunas competencias vinculadas con la autorrealización, la autoinstrucción o la defensa de los propios intereses son difícilmente aprehendibles para las personas con discapacidad intelectual porque involucran procesos de introspección y precisan de una instrucción más explícita que no se adquiere de forma espontánea (Chou et al., 2017). No obstante, a pesar de la dificultad, fortalecer la capacidad de autodefensa de estas personas (expresar opiniones propias, defender los intereses personales, etc.) se convierte en un objetivo prioritario en la lucha contra la desigualdad. De hecho, la propia Convención Internacional sobre los Derechos de las Personas con Discapacidad (ONU, 2006) se refiere a la necesidad de desarrollar estas habilidades para hacer frente a la situación de "doble discriminación” que sufren las personas con discapacidad intelectual: por tener discapacidad y por ser esta intelectual.

A este respecto, si bien existen varias propuestas de intervención sistemáticas para trabajar autodeterminación en la adultez (por ejemplo: Guías de Buenas Prácticas sobre autodeterminación impulsadas por Plena inclusión desde 2010), la mayoría están destinadas a personas adultas-jóvenes que se desenvuelven en un entorno laboral. No obstante, tal y como señalan Oswald et al. (2018), los desafíos a los que se enfrentan las personas con discapacidad durante la edad adulta van más allá del ámbito laboral y requieren nuevas formas de orientar el trabajo sobre autodeterminación. En este sentido, y de acuerdo con Carter et al. (2008), cobra especial relevancia diseñar programas formativos específicos a las necesidades que puedan surgir a lo largo de esta etapa, por ejemplo: duelo por pérdida de personas significativas, reubicación del lugar habitual de residencia, envejecimiento, etc.

Pese a los bajos niveles de autodeterminación evidenciados en este estudio, entre las conductas de los y las participantes se observan con bastante frecuencia algunas competencias relacionadas con la autodeterminación. La realización de elecciones y la resolución de problemas cotidianos son dos de las habilidades que los adultos y adultas con discapacidad en esta investigación llevan a cabo con mayor asiduidad. En estudios como los de Vega et al. (2018) también se aprecian con periodicidad estas conductas. No obstante, autores como Arellano y Peralta (2015) sugieren contrastar 
con las propias personas si las decisiones que toman o los problemas que resuelven son significativos para ellas o responden a las inquietudes de otros. De ser este el caso, no podría hablarse de acciones volitivas ni autodeterminadas porque no tributan a intereses propios.

En esta línea, la literatura sugiere (Chou et al., 2017) que para garantizar la autonomía en los procesos de toma de decisiones es imprescindible trabajar habilidades relacionadas con el autoconocimiento. En esta investigación, al igual que en estudios afines (Chadwick y Fullwood, 2018; Finn et al., 2008; Mumbardó-Adam et al., 2017; Vicente et al., 2017; Wehmeyer et al., 2011), las personas con discapacidad intelectual cuentan con escasas competencias para desarrollar habilidades de autoconocimiento. En este sentido, las conclusiones de investigaciones como las de Ali, Hassiotis, Strydom y King (2012), Álvarez-Aguado, Vega, Spencer, González y Arriagada (2019) y Heller et al. (2011) evidencian cómo profesionales o familias apenas otorgan relevancia al autoconocimiento como factor importante para el logro de la autonomía personal y, en consecuencia, de la autodeterminación. Según estos estudios, es alto el número de profesionales o padres y madres que prefieren no hablar con las propias personas sobre las diferencias que las caracterizan. Este posicionamiento, según Arellano y Peralta (2015), constituye una importante barrera para la inclusión porque dificulta naturalizar la diversidad humana.

Por otra parte, este estudio pone de relieve la importancia que el desarrollo de competencias de autodeterminación tiene para las propias personas con discapacidad. En este sentido, algunos estudios han demostrado que el desempeño de estas habilidades se estanca en torno a los 45 años debido al prematuro proceso de envejecimiento que sufren las personas con discapacidad intelectual (Navas, Uhlmann y Berástegui, 2014; Rodríguez-Aguilella, Verdugo y Sánchez, 2015). Esto supone un gran desafío y pone de relieve la necesidad, tal y como sugieren algunos autores (Ali et al., 2012; Arellano y Peralta, 2015), de involucrar a agentes informales como la familia o la comunidad para apoyar el fortalecimiento de estas habilidades más allá de las aulas. De ahí la idea de entender la autodeterminación como el resultado de una sana interdependencia entre los distintos agentes involucrados.

En esta investigación, y en línea con estudios como los de Arellano y Peralta (2015) o Grigal et al. (2003), familia y profesionales de atención directa se configuran como agentes principales de apoyo al desarrollo de conductas autodeterminadas. No obstante, en ocasiones estos mismos agentes pueden actuar como barreras hacia el constructo si incurren en conductas sobreprotectoras (Jones et al., 2018). A este respecto, las propias personas con discapacidad reconocen que, con frecuencia, sus familias u otros miembros de la comunidad tienden a decidir por ellos y ellas, controlan sus gastos, son intransigentes ante metas u objetivos personales, etc. (Nonnemacher y Bambara, 2011). Precisamente, para garantizar el derecho a la vida independiente, la figura del asistente personal se ha erigido en la actualidad como una de las principales estrategias de apoyo al desarrollo de proyectos de vida elegidos por las propias personas (Plena inclusión, 2017). Sería interesante estudiar con mayor profundidad qué oportunidades ofrece esta nueva figura para potenciar la autodeterminación personal. 
Por otra parte, al igual que estudios como los de Carter et al. (2008) o GómezVela et al. (2012), esta investigación ha evidenciado que factores como la edad o el género son variables no definitorias de autodeterminación. De hecho, los resultados de este estudio, en línea con Wehmeyer et al. (2011), han puesto de manifiesto que algunos elementos de carácter contextual condicionan el desarrollo de la autodeterminación. En este caso, participar activamente en procesos de investigación inclusiva evidencia mayores niveles de autodeterminación, sobre todo a la hora de autoevaluar la propia conducta y establecer metas personales. Estos resultados son coincidentes con las conclusiones de investigaciones como las de Bigby et al. (2014), Pallisera et al. (2017) o Walmsley et al. (2018). Estos estudios hablan de un incremento notable de la satisfacción personal y un mejor manejo de habilidades relacionadas con la autodeterminación (autoevaluación, autoinstrucción, autonomía, etc.) en aquellas personas con discapacidad que forman parte de grupos colaboradores o equipos asesores en los procesos de investigación que les atañen.

Por otro lado, entre los relatos de algunos participantes se alude a la falta de oportunidades para poder ejecutar comportamientos autodeterminados. Esta idea también queda reflejada en los testimonios de personas adultas con discapacidad intelectual que han participado en investigaciones como las de Vega et al. (2018) o Chou et al. (2017). A este respecto, algunos estudios muestran cómo las oportunidades para ejercer la autodeterminación en la adultez se limitan a la mejora de ciertos comportamientos (manejo de conductas disruptivas, por ejemplo) o al desarrollo de algunas habilidades de la vida diaria (Ali et al., 2012; Wehmeyer et al., 2013). No obstante, los propios adultos y adultas con discapacidad van más allá y manifiestan la necesidad de, por ejemplo, participar en la planificación de sus itinerarios de salud, la autogestión de sus procesos de duelo, la elección de las alternativas residenciales más acordes a las propias preferencias, el establecimiento de relaciones sociales significativas, la recepción de cuidados conforme a sus voluntades, creencias religiosas o culturales, etc. En este sentido, en línea con Pallisera et al. (2018) urge ofrecer oportunidades para desarrollar habilidades de autodeterminación desde estas áreas.

Por otra parte, se asume que una de las principales limitaciones de esta investigación radica en la imposibilidad de generalizar los resultados. Sin embargo, este estudio presenta algunas fortalezas que es necesario resaltar. Por un lado, contribuye a enriquecer el grueso de investigaciones sobre autodeterminación en la adultez de personas con discapacidad intelectual, siendo este un aspecto apenas abordado en Latinoamérica. Igualmente, complementa los resultados de otras investigaciones con respecto a los factores que pueden incidir en el desarrollo de estas habilidades a lo largo de la vida; y lo hace a partir de las autopercepciones de las propias personas. Todo esto facilita ir definiendo instancias que proporcionen oportunidades para desarrollar la autodeterminación sin que la etapa vital sea un elemento condicionante.

\section{Referencias bibliográficas}

Abery, B. y Stancliffe, R. J. (2003). A tripartite-ecological theory of self-determination. En M. L. Wehmeyer, B. H. Abery, D. E. Mithaug y R. J. Stancliffe (Eds.), Theory in 
AVANZANDO EN AUTODETERMINACIÓN: ESTUDIO SOBRE LAS AUTOPERCEPCIONES DE PERSONAS

ADULTAS CON DISCAPACIDAD INTELECTUAL DESDE UNA PERSPECTIVA DE INVESTIGACIÓN INCLUSIVA V. VEGA CÓRDOVA, I. ÁLVAREZ-AGUADO, H. SPENCER GONZÁLEZ Y F. GONZÁLEZ CARRASCO

self-determination. Foundations for educational practice (pp. 43-78). Springfield, IL: Charles C. Thomas.

Agran, M., Blanchard, C., Wehmeyer, M. L. y Hughes, C. (2002). Increasing the problemsolving skills of students with developmental disabilities participating in general education. Remedial and Special Education, 23(5), 279-288. https://doi.org/10.1177/07419325020230050301.

Agran, M., Cavin, M., Wehmeyer, M. L. y Palmer, S. (2010). Promoting active engagement in the general education curriculum for students with cognitive disabilities. Education and Training in Autism and Developmental Disabilities, 45(2), 163-174.

Ali, A., Hassiotis, A., Strydom, A. y King, M. (2012). Self stigma in people with intellectual disabilities and courtesy stigma in family carers: A systematic review. Research in Developmental Disabilities, 33(6), 2122-2140. https://doi.org/10.1016/j.ridd.2012.06.013.

Álvarez-Aguado, I., Vega, V., Spencer, H., González, F. y Arriagada, R. (2019). Adultos chilenos con discapacidad intelectual: creencias, actitudes y percepciones parentales sobre su autodeterminación. Siglo Cero, 50(2), 51-72. https://doi.org/10.14201/scero20195025172.

Arellano, A. y Peralta, F. (2015). El Enfoque Centrado en la Familia en el campo de la discapacidad intelectual ¿Cómo perciben los padres su relación con los profesionales? Revista de Investigación Educativa, 33(1), 119-132. https://doi.org/10.6018/rie.33.1.198561.

Bigby, C., Frawley, P. y Ramcharan, P. (2014). A collaborative group method of inclusive research. Journal of Applied Research in Intellectual Disabilities, 27(1), 54-64. https://doi. org/10.1111/jar.12082.

Carter, E. W., Lane, K. L., Pierson, M. R. y Stang, K. K. (2008). Promoting self-determination for transition-age youth: Views of high school general and special educators. Exceptional Children, 75(1), 55-70. https://doi.org/10.1177/001440290807500103.

Castro, L., Casas, J., Sánchez, S., Vallejos, V. y Zúñiga, D. (2016). Percepción de la calidad de vida en personas con discapacidad y su relación con la educación. Estudios Pedagógicos, 42(2), 39-49. https://doi.org/10.4067/S0718-07052016000200003.

Chadwick, D. D. y Fullwood, C. (2018). An online life like any other: identity, self-determination, and social networking among adults with intellectual disabilities. Cyberpsychology, Behavior, and Social Networking, 21(1), 56-64. https://doi.org/10.1089/cyber.2016.0689.

Chou, Y. C., Wehmeyer, M. L., Palmer, S. B. y Lee, J. (2017). Comparisons of self-determination among students with autism, intellectual disability, and learning disabilities: a multivariate analysis. Focus on Autism and Other Developmental Disabilities, 32(2), 124-132. https://doi.org/10.1177/1088357615611391.

Cote, D. L., Jones, V. L., Barnett, C., Pavelek, K., Nguyen, H. y Sparks, S. L. (2014). Teaching problem solving skills to elementary age students with autism. Education and Training in Autism and Developmental Disabilities, 49(2), 189-199.

Finn, D., Getzel, E. E. y McManus, S. (2008). Adapting the self-determined Learning Model of Instruction for college students with disabilities. Career Development for Exceptional Individuals, 31(2), 85-93. https://doi.org/10.1177/0885728808318327.

Frielink, N., Schuengel, C. y Embregts, P. J. (2018). Autonomy support, need satisfaction, and motivation for support among adults with intellectual disability: testing a self-determination theory model. American Journal on Intellectual and Developmental Disabilities, 123(1), 33-49. https://doi.org/10.1352/1944-7558-123.1.33.

Gómez-Vela, M., Verdugo, M. Á., González, F., Badía, M. y Wehmeyer, M. L. (2012). Assessment of the self-determination of spanish students with intellectual disabilities and other educational needs. Education and Training in Autism and Developmental Disabilities, 47(1), 48-57. 
AVANZANDO EN AUTODETERMINACIÓN: ESTUDIO SOBRE LAS AUTOPERCEPCIONES DE PERSONAS

ADULTAS CON DISCAPACIDAD INTELECTUAL DESDE UNA PERSPECTIVA DE INVESTIGACIÓN INCLUSIVA V. VEGA CÓRDOVA, I. ÁlVAREZ-AGUADO, H. SPENCER GONZÁLEZ Y F. GONZÁLEZ CARRASCO

Gray, N., Basualto, C. y Sisto, V. (2017). Política pública sobre capacitación y empleo en Chile: inclusión/exclusión de una fuerza laboral que envejece. Polis. Revista Latinoamericana, 16(48), 81-106. https://doi.org/10.4067/S0718-65682017000300081.

Grigal, M., Neubert, D., Moon, M. y Graham, S. (2003). Self-determination for students with disabilities: views of parents and teachers. Exceptional Children, 70(1), 97-112. https:// doi.org/10.1177/001440290307000106.

Heller, T., Schindler, A., Palmer, S., Wehmeyer, M. L., Jenson, R., Abery, B. et al. (2011). Self-determination across the life span: issues and gaps. Exceptionality, 19(1), 35-45. https:// doi.org/10.1080/09362835.2011.537228.

Hsien, H. F. y Shannon, S. E. (2005). Three approaches to qualitative content analysis. Qualitative Health Research, 15(9), 1277-1288. https://doi.org/10.1177/1049732305276687.

Jones, J. L., Shogren, K. A., Grandfield, E. M., Vierling, K. L., Gallus, K. L. y Shaw, L. A. (2018). Examining predictors of self-determination in adults with intellectual and developmental disabilities. Journal of Developmental and Physical Disabilities, 1-14. https:// doi.org/10.1007/s10882-018-9607-z.

Kim, N. H. y PARK, J. (2012). The effects of the family-involved SDLMI on academic engagement and goal attainment of middle school students with disabilities who exhibit problem behavior. International Journal of Special Education, 27(1), 117-127. https://doi. org/10.1177/0022466907312354.

Kleinert, J. O., Harrison, E., Dueppen, B. M., Mills, K. R. y Trailor, A. M. (2014). Selfdetermined goal selection and planning by students with disabilities across grade bands and disability categories. Education and Training in Autism and Developmental Disabilities, 49(3), 464-477.

Lee, S-H., Wehmeyer, M. L., Palmer, S. B., Soukup, J. H. y Little, T. D. (2008). Self-determination and access to the general education curriculum. The Journal of Special Education, 42(2), 91-107.

Ley 20.422/2010 que Establece Normas sobre Igualdad de Oportunidades e Inclusión Social de Personas con Discapacidad. Ministerio de Planificación. Gobierno de Chile.

Mella, S., Díaz, N., Muñoz, S., Orrego, M. y Rivera, C. (2014). Percepción de facilitadores, barreras y necesidades de apoyo de estudiantes con discapacidad en la Universidad de Chile. Revista Latinoamericana de Educación Inclusiva, 8(1), 63-80.

Mumbardó-Adam, C., Vicente, E., Giné, C., Guàrdia-Olmos, J., Raley, S. K. y Verdugo, M. Á. (2017). Promoviendo la autodeterminación en el aula: el Modelo de Enseñanza y Aprendizaje de la Autodeterminación. Siglo Cero, 48(2), 41-59. https://doi.org/10.14201/ scero20174824159.

Navas, P., Uhlmann, S. y Berástegui, A. (2014). Envejecimiento activo y discapacidad intelectual. Madrid: Ministerio de Educación, Cultura y Deporte.

Nonnemacher, S. L. y Bambara, L. M. (2011). "I'm supposed to be in charge": self-advocates' perspectives on their self-determination support needs. Intellectual and Developmental Disabilities, 49(5), 327-340. https://doi.org/10.1352/1934-9556-49.5.327.

Nota, I., Ferrari, L., Soresi, S. y Wehmeyer, M. L. (2007). Self-determination, social abilities and the quality of life of people with intellectual disabilities. Journal of Hapiness Study, 12, 245-266. https://doi.org/10.1111/j.1365-2788.2006.00939.x.

Organización de Naciones Unidas (2006). Convención Internacional sobre los Derechos de las Personas con Discapacidad.

Oswald, T. M., Winder-Patel, B., Ruder, S., Xing, G., Stahmer, A. y Solomon, M. (2018). A pilot randomized controlled trial of the ACCESS Program: a group intervention 
AVANZANDO EN AUTODETERMINACIÓN: ESTUDIO SOBRE LAS AUTOPERCEPCIONES DE PERSONAS

ADULTAS CON DISCAPACIDAD INTELECTUAL DESDE UNA PERSPECTIVA DE INVESTIGACIÓN INCLUSIVA V. VEGA CÓRDOVA, I. ÁLVAREZ-AGUADO, H. SPENCER GONZÁLEZ Y F. GONZÁLEZ CARRASCO

to improve social, adaptive functioning, stress coping, and self-determination outcomes in young adults with autism spectrum disorder. Journal of Autism and Developmental Disorders, 48(5), 1742-1760. https://doi.org/10.1007/s10803-017-3421-9.

Pallisera, M., Fullana, J., Puyaltó, C., Vilà, M. y Díaz, G. (2017). Apoyando la participación real de las personas con discapacidad intelectual: una experiencia de investigación inclusiva sobre vida independiente. Revista Española de Discapacidad, 5(1), 7-24. https:// doi.org/10.5569/2340-5104.

Pallisera, M., Fullana, J., Puyaltó, C., Vilà, M., Valls, M. J., Díaz, G. et al. (2018). Retos para la vida independiente de las personas con discapacidad intelectual: un estudio basado en sus opiniones, las de sus familias y las de los profesionales. Revista Española de Discapacidad, 6(1), 7-29. https://doi.org/10.5569/2340-5104.06.01.01.

Pedrosa, I., SuÁrez, J. y García, E. (2013). Evidencias sobre la validez de contenido: avances teóricos y métodos para su estimación. Acción Psicológica, 10(2), 3-20.

Pérez-Llantada, M. C. y López, A. (2004). Metodología de encuestas: conceptos básicos y diseños. En S. Fontes de Gracia, C. García, A. J. Garriga, M. C. Pérez-Llantada y E. Sarriá (Eds.), Diseños de investigación en psicología (3. ${ }^{a}$ ed.), (pp. 433-468). Madrid: Lerko Print.

Petri, J., Embregts, P. J., Taminiau, E. F., Heerkens, L., Schippers, A. P. y Van Hove, G. (2018). Collaboration in inclusive research: competencies considered important for people with and without intellectual disabilities. Journal of Policy and Practice in Intellectual D $i$ sabilities, 15(3), 193-201.

Plena inclusión (2017). Asistencia personal: una herramienta clave para el ejercicio del derecho a la vida independiente de las personas con discapacidad intelectual o del desarrollo. Madrid: Plena inclusión España.

Rodríguez-Aguilella, A., Verdugo, M. Á. y Sánchez, M. C. (2015). Calidad de vida familiar y apoyos para los progenitores de personas con discapacidad intelectual en proceso de envejecimiento. Siglo Cero, 39(227), 19-34.

Salmon, N., Barry, A. y Hutchins, E. (2018). Inclusive research: an irish perspective. British Journal of Learning Disabilities, 46(4), 268-277.

SAndín, M. P. (2003). Investigación cualitativa en educación. Fundamentos y tradiciones. Madrid: McGraw-Hill.

Schalock, R. L., Keith, K. D., Verdugo, M. Á. y Gómez, L. (2010). Quality of life model development and use in the field of intellectual disability. En R. Kober (Ed.), Enhancing the quality of life of people with intellectual disabilities (pp. 17-32). Nueva York: Springer.

Shogren, K. A., Wehmeyer, M. L., Palmer, S. B., Forber-Pratt, A. J., Little, T. D. y LóPEZ, S. (2015a). Causal agency theory: reconceptualizing a functional model of self-determination. Education and Training in Autism and Developmental Disabilities, 50, 251-263.

Shogren, K. A., Wehmeyer, M. L., Palmer, S. y Paek, Y. (2013). Exploring personal and school environment characteristics that predicts self-determination. Exceptionality: a Special Education Journal, 21(3), 147-157. https://doi.org/10.1080/09362835.2013.802231.

Shogren, K. A., Wehmeyer, M. L., Palmer, S. B., Rifenbark, G. G. y Little, T. D. (2015b). Relationships between self-determination and postschool outcomes for youth with disabilities. The Journal of Special Education, 48(4), 256-267. https://doi. org/10.1177/0022466913489733.

Strnadová, I., Cumming, T. M., Knox, M. y Parmenter, T. (2014). Welcome to our class research group. Building an inclusive research team: the importance of team building and skills training. Journal of Applied Research in Intellectual Disabilities, 27(1), 13-22. https:// doi.org/10.1111/jar.12076.

Ediciones Universidad de Salamanca / CC BY-NC-ND

Siglo Cero, vol. 51 (1), 2020, enero-marzo, pp. 31-52 
AVANZANDO EN AUTODETERMINACIÓN: ESTUDIO SOBRE LAS AUTOPERCEPCIONES DE PERSONAS

ADULTAS CON DISCAPACIDAD INTELECTUAL DESDE UNA PERSPECTIVA DE INVESTIGACIÓN INCLUSIVA V. VEGA CÓRDOVA, I. ÁLVAREZ-AGUADO, H. SPENCER GONZÁLEZ Y F. GONZÁLEZ CARRASCO

Tristán, A. (2008). Modificación al modelo de Lawshe para el dictamen cuantitativo de la validez de contenido de un instrumento objetivo. Avances en Medición, 6(1), 37-48.

Vega, V., Álvarez-Aguado, I. y Jenaro, C. (2018). Autodeterminación: explorando las autopercepciones de adultos con síndrome de Down chilenos. Siglo Cero, 49(2), 89-104. https://doi.org/10.14201/scero201849289104.

Vega, V., Jenaro, C., Flores, N., Cruz, M. y Lerdo, A. (2012). Necesidades de apoyos de adultos con discapacidad intelectual institucionalizados en Chile, desde la perspectiva de los proveedores de servicios. Avances en Psicología Latinoamericana, 30, 137-145. https:// doi.org/10.15332/s1794-9998.2012.0002.01.

Vega, V.,Jenaro, C., Morillo, M. L., Cruz, M. y Flores, N. (2011). Calidad de vida y apoyos en personas con discapacidad intelectual institucionalizados en Chile: estudio piloto. Psicología, Conocimiento y Sociedad, 1(3), 923-932. https://doi.org/10.11144/Javeriana.UPSY12-3.cvad.

Verdugo, M. Á., Vicente, E., Gómez-Vela, M., Fernández-Pulido, R., Wehmeyer, M. L., BADIA, M. et al. (2015). Escala ARC-INICO de Evaluación de la Autodeterminación. Manual de aplicación y corrección. Salamanca: INICO.

Vicente, E., Guillén, V., Verdugo, M. Á. y Calvo, I. (2018). El rol de los factores personales y familiares en la autodeterminación de jóvenes con discapacidad intelectual. Educational Psychology, 24(2), 75-83. https://doi.org/10.5093/psed2018a13.

Vicente, E., Verdugo, M. Á., Gómez-Vela, M., Fernández-Pulido, R., Wehmeyer, M. L. y Guillén, V. (2017). Personal characteristics and school contextual variables associated with student self-determination in spanish context. Journal of Intellectual and Developmental Disability, 44(1), 23-34. https://doi.org/10.3109/13668250.2017.1310828.

Walmsley, J. y Johnson, K. (2003). Inclusive research with people with learning disabilities: past, present and futures. Leeds: Jessica Kingsley Publishers.

Walmsley, J., Strnadová, I. y Johnson, K. (2018). The added value of inclusive research. Journal of Applied Research in Intellectual Disabilities, 31(5), 751-759.

Wehmeyer, M. L. (1995). The Arc's Self-Determination Scale: Procedural Guidelines.

Wehmeyer, M. L. (1999). A functional model of self-determination: describing development and implementing instruction. Focus on Autism and Other Developmental Disabilities, 14(1), 53-61. https://doi.org/10.1177/108835769901400107.

Wehmeyer, M. L., Abery, B., Zhang, D., Ward, K., Willis, D., Amin Hossain, W. et al. (2011). Personal self-determination and moderating variables that impact efforts to promote self-determination. Exceptionality: A Special Education Journal, 19(1), 19-30. https://doi. org/10.1080/09362835.2011.537225.

Wehmeyer, M. L., Little, T. D. y Sergeant, J. (2009). Self-determination. En S. J. López, y C. R. SNyder (Eds.), Oxford handbook of positive psychology (2. $\left.{ }^{\mathrm{a}} \mathrm{ed}.\right)$, (pp. 357-366). Nueva York: Oxford University Press.

Wehmeyer, M. L., Palmer, S. B., Shogren, K., Williams-Diehm, K. y Soukup, J. H. (2013). Establishing a causal relationship between intervention to promote self-determination and enhanced student self-determination. The Journal of Special Education, 46(4), 195-210. https://doi.org/10.1177/0022466910392377.

Wehmeyer, M. L., Shogren, K. y Thompson, J. (2018). Self-determination and adult transitions and supports. New directions for adult and continuing education, 160, 56-62. https:// doi.org/10.1002/ace.20299.

Ediciones Universidad de Salamanca / CC BY-NC-ND

Siglo Cero, vol. 51 (1), 2020, enero-marzo, pp. 31-52 